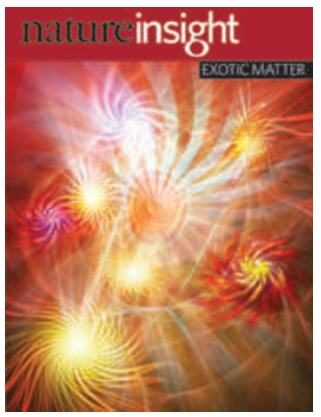

Cover illustration Artwork by N. Spencer

\section{Editor, Nature}

Philip Campbell

Publishing

Nick Campbell

Claudia Deasy

Insights Editor

Karl Ziemelis

\section{Production Editor}

Davina Dadley-Moore

Senior Art Editor

Martin Harrison

Art Editor

Nik Spencer

Sponsorship

Amélie Pequignot

Reya Silao

Production

Jocelyn Hilton

Marketing

Elena Woodstock

Emily Elkins

Editorial Assistant

Emma Gibson

\title{
EXOTIC MATTER
}

REVIEW

176 The enigma of supersolidity

S. Balibar

we might think about day to day. But

these are not the only phases into which

matter can be classified. Consider, for instance, metals, antiferromagnets, ferroelectrics, liquid crystals and

Bose-Einstein condensates or the many solid phases into which liquids can crystallize.

There are numerous ways in which a large number of particles can be ordered, whether the particles are fundamental (such as electrons) or composite (such as atoms and molecules). This ordering is determined by the quantum nature, mutual interactions and symmetries that govern the particles. Crucially, the macroscopic properties of matter usually differ substantially from those of its microscopic constituents. In fact, numerous complex many-body systems show emergent phenomena that are associated with the 'whole' but that cannot be understood solely in terms of the fundamental laws that govern their microscopic 'parts. This complexity is perhaps best encapsulated in Philip Anderson's 'more is different' idea, from his often-quoted 1972 Science article, and is part of the challenge and the beauty of condensed-matter physics.

In this Insight, we expose examples of matter for which 'more is different' holds true in striking ways. We venture into the enigmatic world of supersolids and superconductors, as well as into the frustrated landscapes inhabited by spin liquids and spin ice. We take a look at the one-dimensional confines of nanowires and carbon nanotubes, where correlated electrons form liquids and solids with unique properties that are determined by their low dimensionality. And we dive deep into the exotic realms of topological insulators and non-Abelian states of matter, where quantum effects come with a twist.

We hope that these articles provide a window onto the world of many-body physics, which controls myriad aspects of the world around us, and we thank the authors and reviewers for their invaluable contributions.

Dan Csontos, Associate Editor

\section{PERSPECTIVE}

183 Superconductivity gets an iron boost

I. I. Mazin

\section{REVIEW}

187 Non-Abelian states of matter

A. Stern

\section{PERSPECTIVE}

194 The birth of topological insulators

J. E. Moore

\section{REVIEWS}

199 Spin liquids in frustrated magnets

L. Balents

209 Electron liquids and solids in one dimension

V. V. Deshpande, M. Bockrath, L. I. Glazman \& A. Yacoby

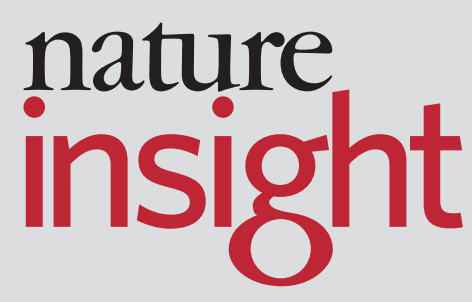

